\title{
Keyword Index Volume 15 (2011)
}

\begin{tabular}{|c|c|c|c|}
\hline \multicolumn{2}{|l|}{ active learning } & data mining techniques & 413 \\
\hline \multicolumn{2}{|l|}{ activity recognition } & data quality & 749 \\
\hline \multicolumn{2}{|l|}{ acute leukemia classification } & data stream & 571,655 \\
\hline agent modeling & 131 & data transformation & 613 \\
\hline aggregated data & 109 & decision tree & 173,671 \\
\hline & 955 & density based clustering & 343 \\
\hline $\begin{array}{l}\text { alert correlation } \\
\text { anomaly detection }\end{array}$ & 151 & density estimation & 343 \\
\hline approximate weighted support & 551 & dependability & 801 \\
\hline association rule & 173,749 & dimensionality reduction & 733 \\
\hline attribute construction & 613 & Dirichlet distribution & 151 \\
\hline \multirow[t]{2}{*}{ attribute selection } & 715 & distributed data streams & 3 \\
\hline & & distributed datasets & 503 \\
\hline back propagation network & 763 & D-S evidence theory & 399 \\
\hline bankruptcy & 237 & & \\
\hline Bayesian multi-nets & 955 & EM algorithm & 357 \\
\hline Bayesian Network & $151,279,931$ & error detection & 749 \\
\hline behavior distance & 827 & European social survey & 533 \\
\hline belief convergence & 399 & evidence conflict & 399 \\
\hline binary classification & 215 & exploration-exploitation tradeoff & 913 \\
\hline bioinformatics & 861 & & \\
\hline Business Analytics and Optimization & 1001 & $\begin{array}{l}\text { False-Positive Anomaly } \\
\text { feature extraction }\end{array}$ & $\begin{array}{l}931 \\
443\end{array}$ \\
\hline cells images & 443 & feature selection & 375,733 \\
\hline change detection & 655 & financial time series & 779 \\
\hline class imbalance & 215 & forecasting & 763 \\
\hline class noise & 215 & frequent items monitoring & 3 \\
\hline classification & $533,671,715$ & frequent patterns & 571 \\
\hline classifiers & 279 & future trends & 1001 \\
\hline clustering & $357,465,827$ & fuzzy linear regression & 763 \\
\hline collaborative clustering & 49 & & \\
\hline concept drift & 589,655 & genetic algorithm & 357 \\
\hline Continuous Hidden Markov Model & 779 & global $\mathrm{CO} 2$ concentration & 763 \\
\hline correlation & 375 & gradual drift & 589 \\
\hline credit rating & 237 & grouping of cases & 299 \\
\hline data cleaning & 749 & Haar wavelet transform (HWT) & 503 \\
\hline data mining $193,443,571,671,74$ & $49,889,1001$ & heat transfer & 733 \\
\hline
\end{tabular}


1026

hierarchical classification

hierarchical clustering

highly imbalanced classification

IDSs' reliability

imbalanced classes

incremental discretization

incremental mining

induction

influence degree prediction

information diffusion model with time-delay 633

information fusion

inseparability

instance selection

intrusion detection

KDD Cup '99 data set

KDD methods

knowledge representation

K-Winner machine

lazy learning

learning vector quantization

machine learning

machine vision

market basket

Markov games

maximum likelihood

measure theory

memory space complexity

Methodological Research

methodology

metric space

mining software engineering data

mixture model

mobile computing

mode seeking

multiagent reinforcement learning

multigram

multilabel classification

multi-label examples

multiple responses

multi-way data clustering

589
861,979 Nash equilibrium points 913

533 Neural Network 931

319 node ranking 633

nonlinear $\quad 319$

$955 \quad$ Non-Monotonic Reasoning 931

843 non-stationary distribution 193

889 online adaptive clustering 3

671,843 online learning 589

633 open-source platform 671

399 outliers 357

319 overlapping 319

251,801 parameter learning 633

particle swarm optimization $\quad 49$

251 PIPs detection 779

413 plan recognition 131

889 polynomial regression 733

193 preprocessing 613

privacy preserving clustering (PPC) 503

715 privacy preserving data mining (PPDM) 503

237 privacy restrictions 49

probabilistic model 151

251,375, 801 probabilistic segmentation 483

$443 \quad$ Protein Function Prediction 979

749

913 Radio Frequency Identification - RFID 931

357 Real-life Applications 1001

109 reasoning with uncertain evidence 955

299 regression 375

1001 reject option 955

251 reusable components 671

109 robust clustering 357

413 robustness 801

357 rough set 465

343 scaling data perturbation (SDP) 503

913 search constraint 173

483 semi-Naive Bayes models 299

861 semi-supervised learning 655

843 sensor networks 3

533 sequence segmentation 483

695 sequential patterns $\quad 889$

set computation $\quad 889$ 
severe attack prediction

similarity

sliding window

social network analysis

software analysis

stability

statistical learning theory

stepwise regression

stream mining

subgroup discovery

subgroup scoring

subspace identification

summarization

supervised classification

supervised learning

SVM
955 symbolic data

571 text categorization

843

633 tolerance factor

551

483

237

193

733

571

533

533

695

613

299

279

319 transition matrix

237

trend forecasting

779

ubiquitous knowledge discovery $\quad 49$

unsupervised metalearning

827

user modeling

variable kernel

343

variable precision rough set model

465

weighted approximate sequential pattern

551

wireless networks 